\title{
Anxiety and depression in women and men from early pregnancy to 3 -months postpartum
}

\author{
Bárbara Figueiredo • Ana Conde
}

Received: 29 November 2010 / Accepted: 24 March 2011 /Published online: 9 April 2011

(C) Springer-Verlag 2011

\begin{abstract}
To investigate high-anxiety and depression in women and men from early pregnancy to 3-months postpartum, 260 Portuguese couples $(N=520)$ filled in the State-Anxiety Inventory (STAI-S) and the Edinburgh PostNatal Depression Scale (EPDS) at the first, second, and third pregnancy trimesters, childbirth, and 3-months postpartum. Rates for high-anxiety (STAI-S $\geq 45$ ) in women $(13.1 \% ; 12.2 \% ; 18.2 \% ; 18.6 \% ; 4.7 \%)$ and men $(10.1 \%$; $8.0 \%, 7.8 \% ; 8.5 \% ; 4.4 \%$ ) and for depression (EPDS $\geq 10$ ) in women $(20.0 \%, 19.6 \%, 17.4 \%, 17.6 \% ; 11.1 \%)$ and men $(11.3 \% ; 6.6 \% ; 5.5 \% ; 7.5 \% ; 7.2 \%)$ were high. Rates for depression were higher than rates for high-anxiety only in women during early pregnancy and the postpartum, but not at the third pregnancy trimester and childbirth. Rates for high-anxiety and depression were higher in women than in men during pregnancy/childbirth, but not at 3-months postpartum. Rates for high-anxiety but not rates for depression were higher during pregnancy/childbirth compared to 3-months postpartum and only in women. Considering that $15.9 \%$ of the parents-to-be were highly anxious and/or depressed during pregnancy - comparing to 9.3\% at 3-months postpartum - particular attention should be drawn to both women's and men's mental health early in pregnancy.
\end{abstract}

Keywords Anxiety · Childbirth · Depression · Postpartum · Pregnancy

B. Figueiredo $(\bowtie) \cdot$ A. Conde

School of Psychology, University of Minho,

Campus de Gualtar,

4710-057 Braga, Portugal

e-mail: bbfi@psi.uminho.pt

\section{Introduction}

High psychiatric morbidity, generally postpartum depression in women, has been traditionally reported in the literature. However, some studies conducted during the past few years pointed out that high-anxiety is more prevalent than depression either before and after childbirth in women (Lee et al. 2007; Wenzel et al. 2003, 2005). And that highanxiety and depression are more prevalent in pregnancy than during the postpartum period, both in women (Andersson et al. 2006; Breitkopf et al. 2006; Heron et al. 2004; Limlomwongse and Liabsuetrakul 2006) and men (Buist et al. 2003; Condon et al. 2004). Women usually show more high-anxiety (Skari et al. 2002) and depression (Edhborg et al. 2005; Matthey et al. 2000; Perren et al. 2005) than their partners, but only recently, men were included in the studies and some controversial results have appeared in the literature (Field et al. 2006).

Empirical studies reported that more than $25 \%$ of women (Lee et al. 2007; Ross and McLean 2006) and 20\% of their partners (Boyce et al. 2007; Buist et al. 2003; Condon et al. 2004) showed high-anxiety during pregnancy. Both in women as in men, anxiety levels seem to be higher in the first and third trimesters, compared to the second pregnancy trimester (Huizink et al. 2004; Lee et al. 2007; Teixeira et al. 2009). These levels are also higher during pregnancy compared with the postpartum, in women (Andersson et al. 2006; Breitkopf et al. 2006; Heron et al. 2004; Lee et al. 2007) as well as men (Buist et al. 2003; Condon et al. 2004). More women than their partners have been reporting high-anxiety, either before (Dulude et al. 2002) or after childbirth (Skari et al. 2002).

Over $15 \%$ of the pregnant women (Dietz et al. 2007; Figueiredo et al. 2007; Kammerer et al. 2009; Limlomwongse and Liabsuetrakul 2006), but less than $10 \%$ of their partners 
(Buist et al. 2003; Condon et al. 2004; Edhborg et al. 2005; Perren et al. 2005) are depressed antenatally. Rates for depression decrease in women from the first to the third pregnancy trimester (Felice et al. 2004), and from pregnancy to the postpartum period (Field et al. 2008; Kammerer et al. 2009; Limlomwongse and Liabsuetrakul 2006; Perren et al. 2005). Higher rates of depression before childbirth as compared to after were reported also in men (Condon et al. 2004; Perren et al. 2005), but this decrease was not as clear as in women (Buist et al. 2003; Deater-Deckard et al. 1998; Morse et al. 2001). Some studies have however reported higher rates of depression after delivery than during pregnancy in women (Areias et al. 1996; Dietz et al. 2007; Gorman et al. 2004). Moreover, in some studies, postpartum depression was almost as prevalent in men as in women (Dulude et al. 2002; Field et al. 2006). Even so, most studies have been showing two to three times more postnatal depressed mothers than fathers (Areias et al. 1996; Edhborg et al. 2005; Matthey et al. 2000; Morse et al. 2001).

Co-morbid anxiety and depression in women is common during pregnancy and the postpartum period (Andersson et al. 2006; Field et al. 2008; Heron et al. 2004; Wenzel et al. 2005); however there are no available data concerning men.

Research has been giving us some controversial and partial views that we would like to broaden with this study, investigating simultaneously both: anxiety and depression, women and men, and from early pregnancy (first, second, and third trimesters) to childbirth and the postpartum period. This study was aimed to investigate high-anxiety and depression throughout pregnancy (first, second, and third trimesters) and the first postpartum months (childbirth and 3-months postpartum), analyzing differences in rates between (1) highanxiety and depression, (2) women and men, and (3) pregnancy and the postpartum period. To the best of our knowledge, highanxiety and depression have not been investigated together and simultaneously in women and men in a longitudinal study from early pregnancy to the postpartum period.

The study attempts to answer the following three questions which have obtained some mixed results in the literature. (1) Is high-anxiety more or less prevalent than depression during pregnancy and the postpartum period? This question seems relevant taking into account that traditionally most researchers have focused on depression, mainly postpartum depression, while more recently, authors argue the need to better deal with anxiety (Heron et al. 2004; Lee et al. 2007; Wenzel et al. 2003, 2005). (2) Are high anxiety and depression more frequent in women than in men during pregnancy and the postpartum? This is a relevant question in order to clarify if sex differences reported in adult psychological morbidity are still present during pregnancy and the postpartum period. And, to determine if pregnancy and the postpartum are associated with high psychological morbidity in men as well, as pointed out in the recent literature (Boyce et al. 2007; Condon et al. 2004; Dulude et al. 2002; Field et al. 2006) and traditionally argued for women. Is high-anxiety and depression more prevalent during pregnancy or the postpartum? This question is relevant in order to support more recent data, controversial to traditional literature, associating pregnancy and not the postpartum period with higher psychological morbidity, in both women (Andersson et al. 2006; Breitkopf et al. 2006; Limlomwongse and Liabsuetrakul 2006) and men (Buist et al. 2003; Condon et al. 2004). To determine if higher risk for psychological disorder is during pregnancy or during the postpartum period will allow mobilizing preventive and intervening strategies more adequate in timing.

\section{Methods}

Procedures

This research received previous approval from the institution's Ethical Review Board and was conducted in accordance with the Helsinki Declaration. Three hundred pregnant women were randomly recruited in a Public Hospital Obstetrics Out-patients Unit (Porto, Portugal), at their first appointment. The exclusion criteria were: not reading or writing Portuguese and multiple gestations. The aims and the procedures of the study were explained and $84 \%$ of the pregnant women accepted to participate and signed an informed consent. The partner also agreed, signed an informed consent, and was available to participate in $86 \%$ of the cases. The sample of the further analyses is composed by 260 women and their partner $(N=520)$ who both agree to participate in the study.

The socio-demographic questionnaire was separately administered to the pregnant woman and her partner, and the State-Anxiety Inventory (STAI-S) and Edinburgh PostNatal Depression Scale (EPDS) was filled in (between weeks 8 and 14). The STAI-S and EPDS were administrated again separately at the hospital while they were attending their appointment during the second (between weeks 20 and 24) and third pregnancy trimester (between weeks 30 and 34), as well as after childbirth (between days 1 and 3), and were separately mailed at 3-months postpartum (between weeks 10 and 14).

\section{Measures}

Information about the participants (e.g., age, occupational status, marital status) was collected using a Socio-Demographic Questionnaire. 
The STAI-S was used to measure anxiety (Spielberger et al. 1983). The STAI-S is a 20-item self-report scale for measuring the temporary condition of state-anxiety (anxiety in a specific situation). STAI-S Portuguese version has shown good internal consistency (Cronbach alpha $=0.87-$ 0.93). The authors advise a score equal or higher than 45 (STAI-S $\geq 45$ ) to screen for high-anxiety states (focusing in the prevalence of state-anxiety symptoms independently of the related probable anxiety diagnoses), in both women and men (Biaggio et al. 1976).

The Portuguese version of the EPDS (Cox et al. 1987), a self-report questionnaire composed by ten items, was used to address symptoms of depression within the previous 7 days. EPDS Portuguese version has shown good internal consistency (Cronbach Alpha $=0.85$; Figueiredo et al. 2007). In women, a mean EPDS score of 10 was pointed out for 'caseness' of depression-major, minor or intermittent depressive disorder diagnose in the Schedule for Affective Disorders and Schizophrenia interview, with values of sensitivity $=65$ and of specificity $=96$ (Areias et al. 1996). Although scores did not appear to be so sensitive to depression in men, the same cut-off was suggested in the EPDS Portuguese validation, with values of sensitivity $=40$ and of specificity $=81$ (Areias et al. 1996). Thus, a score equal or higher than 10 (EPDS $\geq 10$ ) was used in both women and men to screen for depression (focusing on its prevalence independently of the related probable depression diagnoses).

\section{Data analysis}

McNemar test for two-related samples was used to test significant differences between the proportions of STAI-S $\geq$ 45 and EPDS $\geq 10$. Pearson chi-square and odds ratio statistics were applied to analyze the association between sex and the prevalence of STAI-S $\geq 45$, EPDS $\geq 10$, STAI$\mathrm{S} \geq 45^{\wedge} \mathrm{EPDS} \geq 10$ (meaning that the participant had simultaneously STAI-S total scores equal or higher than 45 and EPDS total scores equal or higher than 10), and STAI-S $<$ $45^{\wedge}$ EPDS $<10$ (meaning that the participant had simultaneously STAI-S total scores lower than 45 and EPDS total scores lower than 10). Cochran's $Q$ statistic for $K$-related samples was used to examine differences between moments of assessment taking into account the prevalence of STAI$\mathrm{S} \geq 45, \quad \mathrm{EPDS} \geq 10, \quad \mathrm{STAI}-\mathrm{S} \geq 45^{\wedge} \mathrm{EPDS} \geq 10$, and STAI-S $<$ $45^{\wedge}$ EPDS $<10$. McNemar two-related sample tests were used for pairwise comparisons between each measuring time point: first, second, and third pregnancy trimesters, childbirth and 3-months postpartum. Since ten groups of comparison were involved, Bonferroni correction (significant, $0.05 / 10$ comparison pairs) needed to be applied, and consequently results are reported at a 0.005 level of significance.

\section{Results}

Participants' socio-demographics

Nearly all the participants were Portuguese (92.1\%), Caucasian (94.8\%) and Catholic (90.6\%). More than half of the women $(63.6 \%)$ and men $(62.9 \%)$ were parents for the first time and aged between 20 and 39 years old. The majority of participants were from a low or medium-low socio-economic level; were employed and had been employed for more than 5 years $(50.0 \%$ of the women and $62.5 \%$ of the men), in manual (qualified or not qualified) professions. The majority of participants were married $(55.1 \%)$ or cohabiting $(33.1 \%)$, only $11.4 \%$ were single and $0.4 \%$ divorced (see Table 1 ).

Only $1.6 \%$ of the participants reported having been hospitalized in a psychiatric hospital, but $26.8 \%$ had taken medication for psychiatric problems, and $11.7 \%$ reported receiving counseling with a psychologist or a psychiatrist.

No significant differences were obtained between women whose partner agreed or did not agree to participate in this study in terms of parity $(\chi 2=0.124 ; p=0.724)$, schooling $(\chi 2=4.086 ; p=0.130)$, profession $(\chi 2=5.605 ; p=0.132)$ and socio-economic level $(\chi 2=5.720 ; p=0.221)$. Significant differences were however obtained concerning women's age $(\chi 2=16.002 ; p=0.001)$ and occupational status $(\chi 2=$ 9.742; $p=0.008)$ : a higher percentage of women whose partners did not agree to participate were younger than 19 years old or older than 40 years old and were unemployed.

Almost all women (91.4\%) and men (82.6\%) completed all five assessments. No socio-demographic significant differences were obtained between women that complete and do not complete all the five assessments concerning to: parity $(\chi 2=2.581 ; p=0.275)$, schooling $(\chi 2=0.547 ; p=$ $0.761)$, socio-economic level $(\chi 2=6.299 ; p=0.178)$, occupational status $(\chi 2=4.615 ; p=0.100)$, and profession $(\chi 2=$ 5.933; $p=0.115)$. No significant differences were also found between men that complete and do not complete all the five assessments related to socio-demographics: parity $(\chi 2=4.956 ; p=0.084)$, schooling $(\chi 2=0.966 ; p=0.617)$, socio-economic level $(\chi 2=7.275 ; p=0.122)$, occupational status $(\chi 2=0.007 ; p=0.996)$, and profession $(\chi 2=7.268$; $p=0.064)$. However, both women $(\chi 2=10.969 ; p=0.012)$ and men $(\chi 2=9.281 ; p=0.026)$ who did not complete all the five assessments were younger than women and men who completed all the assessments.

\section{Anxiety compared to depression}

EPDS $\geq 10$ rates were significantly higher than STAI-S $\geq 45$ rates at the first pregnancy trimester $(\chi 2=6.35, p=0.012)$ 
Table 1 Socio-demographics (women, men and total sample) ${ }^{\mathrm{a}}$

\begin{tabular}{|c|c|c|c|c|}
\hline & & Women $n=260 \%$ & Men $n=260 \%$ & Total $N=520 \%$ \\
\hline \multirow[t]{4}{*}{ Age (years) } & $\leq 19$ & 11.8 & 4.4 & 8.1 \\
\hline & $20-29$ & 46.7 & 39.0 & 42.9 \\
\hline & $30-39$ & 39.5 & 48.2 & 43.9 \\
\hline & $\geq 40$ & 2.0 & 8.4 & 5.1 \\
\hline \multirow[t]{5}{*}{ Socio-economic level } & High & 16.4 & 9.0 & 12.5 \\
\hline & Medium high & 7.4 & 14.3 & 11.1 \\
\hline & Medium & 19.6 & 18.8 & 19.0 \\
\hline & Medium low & 26.5 & 30.1 & 28.6 \\
\hline & Low & 30.1 & 27.8 & 28.8 \\
\hline \multirow[t]{3}{*}{ Occupational status } & Employed & 72.2 & 88.8 & 80.4 \\
\hline & Unemployed & 21.6 & 9.2 & 15.4 \\
\hline & Household/student & 6.2 & 2.0 & 4.2 \\
\hline \multirow[t]{4}{*}{ Profession } & Non manual qualified & 24.1 & 23.4 & 23.7 \\
\hline & Non manual not qualified & 19.3 & 18.8 & 19.0 \\
\hline & Manual qualified & 26.7 & 30.0 & 28.5 \\
\hline & Manual not qualified & 29.9 & 27.8 & 28.8 \\
\hline \multirow[t]{3}{*}{ Schooling (years) } & $<9$ & 26.8 & 40.0 & 33.3 \\
\hline & $9-12$ & 54.7 & 48.4 & 51.6 \\
\hline & $>12$ & 18.5 & 11.6 & 15.1 \\
\hline
\end{tabular}

${ }^{a}$ Available socio-demographics data for childbearing women in the northern area of Portugal: $45.7 \%$ aged between 25 and 29 years are first time parents; $56 \%$ married and $47.3 \%$ living with the partner; $73.3 \%$ employed (INE-Instituto Nacional de Estatística, Portugal, 2001).

and the third postpartum month $(\chi 2=9.48, p=0.002)$, while no significant differences were found at the second $(\chi 2=$ 2.64, $p=0.104)$ and third pregnancy trimesters $(\chi 2=0.68, p=$ $0.410)$, and at childbirth $(\chi 2=0.17, p=0.683$; see Table 2 and Fig. 1).

In women, rates for EPDS $\geq 10$ were significantly higher than rates for STAI-S $\geq 45$ at the first $(\chi 2=7.61, p=0.006)$ and second pregnancy trimesters $(\chi 2=7.23, p=0.007)$, and at 3-months postpartum $(p=0.008)$, but not at the third pregnancy trimester $(\chi 2=0.03, p=0.868)$ or at childbirth $(\chi 2=0.03, p=0.868)$. In men, no significant differences were found between rates of EPDS $\geq 10$ and rates of STAI$\mathrm{S} \geq 45$ at the first $(p=0.690)$, second $(p=0.678)$, and third pregnancy trimesters $(p=0.332)$, neither at childbirth $(p=$ $0.815)$ nor at 3 -months postpartum $(p=0.180$; see Table 2 and Fig. 2).

Table 2 Rates for STAI-S $\geq 45$, EPDS $\geq 10$, STAI-S $\geq 45^{\wedge}$ EPDS $\geq 10$, and STAI-S $<45^{\wedge}$ EPDS $<10$ (in percent, \%) throughout pregnancy and the first postpartum months (women, men and total sample)

\begin{tabular}{|c|c|c|c|c|c|c|}
\hline & & First trimester & Second trimester & Third trimester & Childbirth & 3-months postpartum \\
\hline \multirow[t]{3}{*}{ STAI-S $\geq 45$} & Women & 13.1 & 12.2 & 18.2 & 18.6 & 4.7 \\
\hline & Men & 10.1 & 8.0 & 7.8 & 8.5 & 4.4 \\
\hline & Total & 11.7 & 10.4 & 13.2 & 13.7 & 4.6 \\
\hline \multirow[t]{3}{*}{$\mathrm{EPDS} \geq 10$} & Women & 20.0 & 19.6 & 17.4 & 17.6 & 11.1 \\
\hline & Men & 11.3 & 6.6 & 5.5 & 7.5 & 7.2 \\
\hline & Total & 15.9 & 13.3 & 11.7 & 12.7 & 9.2 \\
\hline \multirow[t]{3}{*}{$\mathrm{STAI}-\mathrm{S} \geq 45^{\wedge} \mathrm{EPDS} \geq 10$} & Women & 9.2 & 7.9 & 10.1 & 9.5 & 3.2 \\
\hline & Men & 5.5 & 2.3 & 2.9 & 3.5 & 3.4 \\
\hline & Total & 7.6 & 5.2 & 6.7 & 6.6 & 3.3 \\
\hline \multirow[t]{3}{*}{ STAI-S $<45^{\wedge}$ EPDS $<10$} & Women & 76.2 & 75.6 & 75.1 & 73.3 & 87.2 \\
\hline & Men & 83.9 & 87.3 & 89.0 & 87.4 & 91.6 \\
\hline & Total & 79.8 & 81.1 & 81.7 & 80.2 & 89.4 \\
\hline
\end{tabular}


Fig. 1 Rates for STAI-S $\geq 45$, EPDS $\geq 10$, STAI-S $\geq 45^{\wedge}$ EPDS $\geq$ 10 , and STAI-S $<45^{\wedge}$ EPDS $<10$ throughout pregnancy and the first postpartum months (total sample). Significant differences are presented with a continuous line

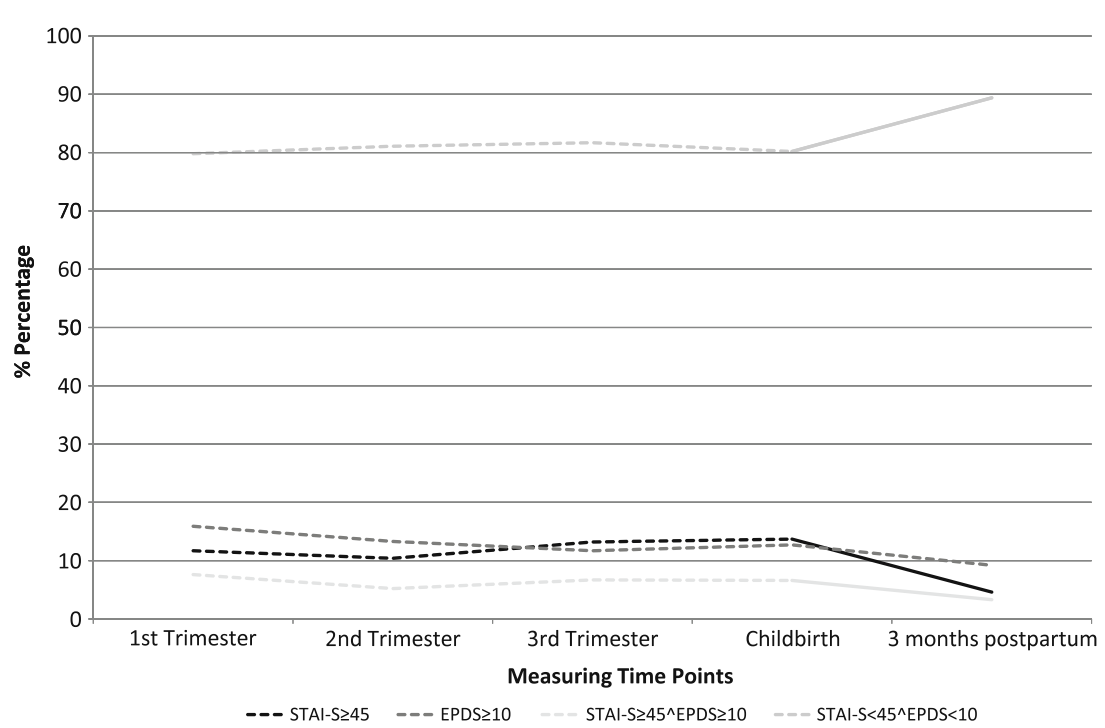

Women compared to men

Significant associations were observed between sex and STAI-S $\geq 45$ rates: at the third pregnancy trimester $(\chi 2=$ $10.75, p=0.001)$ and childbirth $(\chi 2=8.70, p=0.003)$, but not in the first $(\chi 2=1.08, p=0.298)$ and second $(\chi 2=2.35$, $p=0.125)$ pregnancy trimesters, neither at 3 -months postpartum $(\chi 2=0.02, p=0.893)$. According to odds ratio, women were more likely than men to have a STAI-S $\geq 45$ at the third pregnancy trimester $(\mathrm{OR}=2.63 ; 95 \% \mathrm{CI}=1.39$ 3.99) and at childbirth $(\mathrm{OR}=2.44 ; 95 \% \mathrm{CI}=1.28-3.73$; see Table 2 and Fig. 2).

Significant associations between sex and EPDS $\geq 10$ were also obtained in all moments of assessment, except at 3 -months postpartum $(\chi 2=1.63, p=0.202)$. Women were more likely to have an $\operatorname{EPDS} \geq 10$ than men during pregnancy, in the first $(\chi 2=6.98, p=0.008 ; \mathrm{OR}=1.95$; $95 \% \mathrm{CI}=1.14-2.65)$, second $(\chi 2=17.03, p<0.001 ; \mathrm{OR}=$ $3.43 ; 95 \% \mathrm{CI}=1.72-5.17)$, and third trimesters $(\chi 2=15.55$, $p<0.001 ; \mathrm{OR}=3.61 ; 95 \% \mathrm{CI}=1.72-5.88)$, and at childbirth $(\chi 2=9.36, p=0.002 ; \mathrm{OR}=2.67 ; 95 \% \mathrm{CI}=1.33-4.14$; see Table 2 and Fig. 2).

Significant associations were found between sex and rates of $\mathrm{STAI}-\mathrm{S} \geq 45^{\wedge} \mathrm{EPDS} \geq 10$ : in the second $(\chi 2=7.34, p=$ $0.007)$ and third pregnancy trimesters $(\chi 2=9.40, p=0.002)$, as well as at childbirth $(\chi 2=5.98, p=0.009)$, but not at the first pregnancy trimester $(\chi 2=2.48, p=0.115)$ or at 3 -months postpartum $(\chi 2=0.01, p=0.931)$. Significant associations between sex and STAI-S $<45^{\wedge}$ EPDS $<10$ rates were also observed in the first $(\chi 2=4.61, p=0.032)$, second $(\chi 2=$ $10.38, p=0.001)$, and third pregnancy trimester $(\chi 2=14.45$, $p<0.001)$, as well as at childbirth $(\chi 2=12.80, p<0.001)$, but
Fig. 2 Rates for STAI-S $\geq 45$ and $E P D S \geq 10$ throughout pregnancy and the first postpartum months (women and men). Significant differences are presented with a continuous line

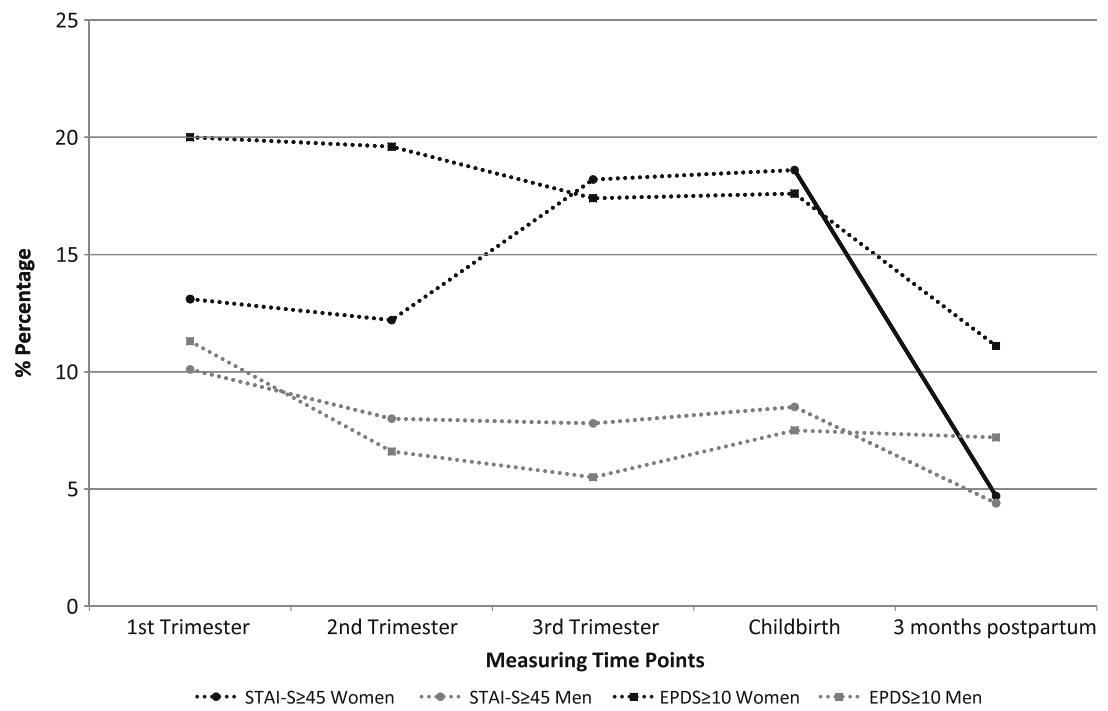


not at 3 -months postpartum $(\chi 2=1.86, p=0.173)$. According to the odds ratio, women were more likely than men to have a STAI-S $\geq 45^{\wedge} \mathrm{EPDS} \geq 10$ in the second $(\mathrm{OR}=3.68 ; 95 \% \mathrm{CI}=$ $1.33-9.22)$, and third trimesters $(\mathrm{OR}=3.83 ; 95 \% \mathrm{CI}=1.49$ 8.58), and at childbirth $(\mathrm{OR}=2.89 ; 95 \% \mathrm{CI}=1.18-6.30)$. While men were more likely than women to have a STAI-S $<$ $45^{\wedge} \mathrm{EPDS}<10$ in the first $(\mathrm{OR}=1.63 ; 95 \% \mathrm{CI}=1.03-2.11)$, second $(\mathrm{OR}=2.22 ; 95 \% \mathrm{CI}=1.25-2.81)$, and third trimesters $(\mathrm{OR}=2.70 ; 95 \% \mathrm{CI}=1.47-3.58)$, and at childbirth $(\mathrm{OR}=$ 2.53; 95\% CI=1.39-3.28; see Table 2).

Pregnancy compared to the first postpartum months

Significant differences were found in participants' rates of STAI-S $\geq 45$ between moments of assessment $[Q(4)=21.39$, $p<0.001]$. Rates of STAI-S $\geq 45$ were lower at 3 -months postpartum than in: the first $(\chi 2=8.31, p=0.004)$, second $(\chi 2=12.12, p<0.001)$, third pregnancy trimesters $(\chi 2=$ 14.88, $p<0.001)$ and childbirth $(\chi 2=16.68, p<0.001$; see Table 2 and Fig. 1). Significant differences in rates of STAI-S $\geq 45$ were found between moments of assessment for women $[Q(4)=24.59, p<0.001]$ : higher in the second ( $p=$ $0.001)$, third pregnancy trimester $(\chi 2=15.75, p<0.001)$, childbirth $(\chi 2=16.53, p<0.001)$, compared to the third postpartum month. No significant differences between moments of assessment were found in men's rates of STAI-S $\geq 45[Q(4)=3.09, p=0.542]$ (see Table 2 and Fig. 2).

No significant differences were found in participant's rates of EPDS $\geq 10$ between moments of assessment $[Q(4)=10.61$, $p=0.108]$. EPDS $\geq 10$ rates were not significantly different between moments of assessment for women $[Q(4)=8.10, p=$ $0.088]$, neither for men $[Q(4)=7.25, p=0.123$ ] (see Table 2 and Fig. 2).

Significant differences were found between moments for STAI-S $\geq 45^{\wedge}$ EPDS $\geq 10$ rates in the total sample $[Q(4)=18.76$, $p=0.001]$, as well as for women $[Q(4)=16.95, p=0.002]$, but not for men $[Q(4)=4.4, p=0.355]$. Rates of STAI-S $\geq$ $45^{\wedge} \mathrm{EPDS} \geq 10$ were lower at the third postpartum month than: at the first $(\chi 2=11.05, p=0.001)$, second $(\chi 2=9.13, p=$ $0.003)$, third pregnancy trimester $(\chi 2=8.49, p=0.004)$ and childbirth $(\chi 2=15.79, p<0.001)$. For women, significant differences were found only between the 3 -months postpartum and: the third pregnancy trimester $(\chi 2=8.76 ; p=0.003)$, and childbirth $(\chi 2=14.05 ; p<0.001)$, being lower at 3-months postpartum (see Table 2).

Significant differences were found in participants' rates of STAI-S $<45^{\wedge}$ EPDS $<10$ across time $[Q(4)=17.55, p=0.002]$, namely for women $[Q(4)=15.92, p=0.003]$, but not for men $[Q(4)=4.40, p=0.355]$. Participants' STAI-S $<45^{\wedge}$ EPDS $<10$ rates increased between the first $(\chi 2=10.40, p=0.001)$, second pregnancy trimester $(\chi 2=9.13, p=0.003)$, childbirth $(\chi 2=15.79, p<0.001)$ and: the third postpartum month. Significant differences were only found in women rates of
STAI-S $<45^{\wedge}$ EPDS $<10$ increasing between childbirth and 3months postpartum $(\chi 2=14.05, p<0.001$; see Table 2$)$.

\section{Discussion}

Supporting high-risk psychiatric morbidity during pregnancy and the postpartum period, both for women's anxiety (Ross et al. 2003; Wenzel et al. 2003, 2005) and depression (Felice et al. 2004; Kammerer et al. 2009; Limlomwongse and Liabsuetrakul 2006; Ross et al. 2004), as well as for men's anxiety (Boyce et al. 2007) and depression (Buist et al. 2003; Dulude et al. 2002; Field et al. 2006), rates for high-anxiety and depression were high during the entire studied period. Furthermore, data also illustrate high co-morbid anxiety and depression either during pregnancy and the postpartum period as expected from literature (Andersson et al. 2006; Field et al. 2008; Heron et al. 2004; Wenzel et al. 2005). Presented results generally show higher rates of depression compared to anxiety, in women compared to men, and during pregnancy compared to 3-months postpartum, as discussed below.

Contrary to findings according to which anxiety is more prevalent than depression during pregnancy and/or the postpartum period (Heron et al. 2004; Lee et al. 2007; Wenzel et al. 2003, 2005), depression was more prevalent than high-anxiety in women either early in pregnancy or at 3-months postpartum. These results may be due to cultural differences, since Portuguese women show higher rates of depression during pregnancy and the postpartum period compared to women from the US and from other European countries (Gorman et al. 2004). Although less usual, higher rates of depression than anxiety disorders in women during pregnancy and the postpartum period were also reported in the literature (Andersson et al. 2006). In addition, results show that depression is more prevalent than anxiety in women but not in men, and depression is not more prevalent than anxiety in women at the third trimester of pregnancy and childbirth.

Women were more likely than men to show high-anxiety at the third pregnancy trimester and at childbirth, but not during early pregnancy or 3 months after childbirth Therefore, highanxiety in women compared to men seems more specific of late pregnancy and childbirth, a result that has been receiving some empirical support in the literature (Dulude et al. 2002). This may be related to the fact that pregnancy/ childbirth particular events are generally perceived as threatening by women (Huizink et al. 2004) and might not be as stressful for men. On the other hand, the first postpartum months do not appear to cause more anxiety in women than in men, pointing out that both women and men may experience similar difficulties in adapting to challenges 
associated with taking care of the infant and the parental role. This may also reflect cultural changes associated to the share of parental responsibilities, between the mother and the father.

Women were more likely than men to be depressed in all of the pregnancy trimesters and at childbirth, but not at 3-months postpartum Higher rates of depression are generally found in women compared to men and does not seem to be specific of the pregnancy period, contrary to what we have discussed regarding high-anxiety during childbirth in mothers. However, in this study the absence of a significant difference in EPDS $\geq 10$ rates between women and men at 3-months postpartum appears specific, and suggests a particular vulnerability for depression in men 3 months after childbirth.

Higher anxiety and depression rates in women than in their partners during pregnancy and at childbirth were also observed in other studies (Edhborg et al. 2005; Matthey et al. 2000; Morse et al. 2001; Skari et al. 2002). Whereas men seemed to be less anxious and depressed than women during the whole pregnancy, at 3-months postpartum they showed rates of high-anxiety and depression as high as their partners in our study. Regarding specifically postpartum depression, although some studies reported higher rates in mothers than in fathers (Areias et al. 1996; Edhborg et al. 2005; Matthey et al. 2000; Morse et al. 2001), our results are in agreement with studies that found similar postpartum depression rates between women and men (Dulude et al. 2002; Field et al. 2006). This also suggests that postpartum depression, a condition that has been mainly associated with the mother's postnatal mood experience, may also be an experience of many fathers. The third postpartum month could also be a difficult period of adaptation for men; while the third pregnancy trimester and childbirth seem to be particularly demanding for women. This may be associated with the challenges of specific developmental tasks differently experienced according to gender. While the preparation to childbirth may be more stressful for mothers, the adjustment to parenting and to the new interactions within the family may be particularly challenging for fathers. Our results support both points the view presented in the literature, as similarities and differences between genders in the adaptation to parenthood (Deater-Deckard et al. 1998; Dulude et al. 2002; Edhborg et al. 2005; Field et al. 2006) were observed. Similarities, for example in the decrease of anxiety and depression from early pregnancy to 3-months postpartum in both women and men; and differences, for example in the significant decrease in anxiety from childbirth to 3-months postpartum observed in women but not in men.

High-anxiety decreased from pregnancy to the postpartum period in women, with lower rates observed at 3-months postpartum Many authors have also recently shown that women's (Andersson et al. 2006; Breitkopf et al. 2006; Heron et al. 2004; Ross et al. 2003) and men's (Boyce et al. 2007; Dulude et al. 2002; Perren et al. 2005) anxiety is higher during pregnancy than in the postpartum period. Additionally, our results clearly pointed out that both women and men are particularly at-risk for high-anxiety during the first pregnancy trimester, suggesting that early gestation developmental tasks (pregnancy acceptance) may be quite difficult for parents-to-be.

Therefore, similar attention should be given to the psychological adjustment of both women and men during pregnancy (Breitkopf et al. 2006; Field et al. 2006), and particularly to the first pregnancy trimester (Buist et al. 2003; Condon et al. 2004), as the attention that has been given to the postpartum period. Pregnancy rather than the postpartum period appear to be the most stressful period for men also (Condon et al. 2004), because men exhibited highest symptoms levels in pregnancy, namely in early pregnancy in some studies (Buist et al. 2003).

At the same time, an improvement was observed in the mental status of both women and men after childbirth. Particularly, women increased their psychological adjustment from childbirth to 3-months postpartum (STAI-S < $45^{\wedge}$ EPDS $<10$ rates augmented while STAI-S $\geq 45^{\wedge}$ EPDS $\geq$ 10 rates decreased), despite some susceptibility to anxiety around childbirth. This might suggest that women and men are stressed in some similar ways while other difficulties are specific to gender/parental roles, but they are mainly able to solve and cope positively with the developmental task during this transition, an hypothesis that has been getting empirical support in the literature (Perren et al. 2005). Empirical studies have been reporting that depression and anxiety is less common at postpartum than during pregnancy in women as in men (Andersson et al. 2006; Field et al. 2006; Limlomwongse and Liabsuetrakul 2006; Perren et al. 2005).

Rates of co-morbid anxiety and depression were high, with a decrease from pregnancy to the first postpartum months in the number of participants simultaneously highly anxious and depressed As a result, rates of both were high in our study: $7.6 \%$ of the participants at the first pregnancy trimester had simultaneous high-anxiety and depression and $89.4 \%$ of the participants at 3-months postpartum presented neither anxiety nor depression. This suggests that pregnancy and the postpartum period may be pictured as a period of high-psychiatric morbidity for some women and men, as well as a period of mental health enhancement for most parents, in accordance to what has been proposed (Perren et al. 2005). Both evidences need to be addressed. That some women/men are at high-risk and could be easily identified early in pregnancy as presenting both depression and anxiety. That the majority of women/men are neither 
high-anxious nor depressed and exhibit better psychological functioning 3 months after childbirth than at the beginning of the pregnancy. These results reiterate the need of distinguish psychological symptoms provoked by normal physiological changes, or the presence of a new baby, from the more problematic symptoms developed during pregnancy and postpartum, reflecting the higher vulnerability of some parents to psychological maladjustment (Kammerer et al. 2006).

Some methodological limitations need to be pointed out. First, we must consider the voluntary nature for the participation in the study, which may have led to a selection bias, in the sense that the women/men who agreed to participate may in fact be those who are less anxious and depressed. This constitutes a particular issue for men, as the rate of fathers-to-be who signed the informed consent form was lower when compared to the rate of mothers-to-be. Another constraint is that data was collected by questionnaires, meaning that we must look cautiously at the clinical significance of the presented results. Not all participants who scored above the threshold for probable depression in the EPDS or for probable high-anxiety in the STAI-S have a diagnosis of a disorder. The implications of that for the obtained results need to be mentioned. Rates differences between studies are due to several reasons, namely to probable cultural differences and timing of assessment, and usually, studies that used interviews and clinical diagnoses report lower rates than studies that use screening instruments to report symptomatology (Matthey et al. 2000; O’Hara and Swain 1996). Prevalence estimates are affected by the nature of the assessment method (larger estimates in studies using self-report measures) and by the length of the postpartum period under evaluation (longer periods predict high prevalence; Matthey et al. 2000), as well as by the fact that "the symptom profile of depression in pregnancy is different from the symptom profile of postpartum depression", as suggested by previous studies (Kammerer et al. 2009, p. 139).

The main clinical implications of the presented results need to be attended when screening or intervening in the transition to parenthood: (1) to anxiety and not only to depression; (2) to men and not only women, (3) to pregnancy and not only to the postpartum period. Research and clinical efforts need to be moved towards understanding, recognizing and treating antenatal anxiety/depression, namely identifying the psychosocial and biological bases of antenatal and postnatal psychological (mal)adjustment (Evans et al. 2001; Kammerer et al. 2006).

Acknowledgments This work was supported by the Operational Program Science and Innovation 2010 (POCI 2010) of the Community Support Board III and supported by the European Community Fund FEDER (POCI/SAU-ESP/56397/2004; Anxiety and depression in women and men during the transition to parenthood: effects on fetal and neo-natal behavior and development). We gratefully acknowledge the proofreading of this manuscript by Dra. Gena Rodrigues.

\section{References}

Andersson L, Sundström-Poromaa I, Wulff M, Aström M, Bixo M (2006) Depression and anxiety during pregnancy and six months postpartum: a follow-up study. Acta Obstet Gynecol Scand 85:937-944

Areias ME, Kumar R, Barros H, Figueiredo E (1996) Comparative incidence of depression in women and men, during pregnancy and after childbirth. Br J Psychiatry 169:30-35

Biaggio AM, Natalicio L, Spielberger CD (1976) The development and validation of an experimental Portuguese form of the statetrait anxiety inventory. In: Spielberger CD, Dias-Guerrero R (eds) Cross-cultural research on anxiety. Hemisphere/Wiley, Washington, DC, pp 29-40

Boyce P, Condon J, Barton J, Corkindale C (2007) First-Time Fathers' Study: psychological distress in expectant fathers during pregnancy. Aust N Z J Psychiatry 41:718-725

Breitkopf CR, Primeau LA, Levine RE, Olson GL, Wu ZH, Berenson AB (2006) Anxiety symptoms during pregnancy and postpartum. J Psychosom Obstet Gynaecol 27:157-162

Buist A, Morse CA, Durkin S (2003) Men's adjustment to fatherhood: implications for obstetric health care. J Obstet Gynecol Neonatal Nurs 32:172-180

Condon JT, Boyce P, Corkindale CJ (2004) The First-Time Fathers Study: a prospective study of the mental health and wellbeing of men during the transition to parenthood. Aust $\mathrm{N} \mathrm{Z} \mathrm{J} \mathrm{Psychiatry}$ 38:56-64

Cox JL, Holden JM, Sagovsky R (1987) Detection of postnatal depression. Development of the 10-item Edinburgh postnatal depression scale. Br J Psychiatry 150:782-786

Deater-Deckard K, Pickering K, Dunn JF, Golding J (1998) Family structure and depressive symptoms in men preceding and following the birth of a child. The Avon Longitudinal Study of Pregnancy and Childhood Study Team. Am J Psychiatry 155:818-823

Dietz PM, Williams SB, Callaghan WM, Bachman DJ, Whitlock EP, Hornbrook MC (2007) Clinically identified maternal depression before, during, and after pregnancies ending in live births. Am J Psychiatry 164:1515-1520

Dulude D, Bélanger C, Wright J, Sabourin S (2002) High-risk pregnancies, psychological distress, and dyadic adjustment. J Reprod Infant Psychol 20:101-123

Edhborg M, Matthiesen AS, Lundh W, Widstrom AM (2005) Some early indicators for depressive symptoms and bonding 2 months postpartum-a study of new mothers and fathers. Arch Womens Ment Health 8:221-231

Evans J, Heron J, Francomb H, Oke S, Golding J (2001) Cohort study of depressed mood during pregnancy and after childbirth. BMJ 323:257-260

Felice E, Saliba J, Grech V, Cox J (2004) Prevalence rates and psychosocial characteristics associated with depression in pregnancy and postpartum in Maltese women. J Affect Disord 82:297-301

Field T, Diego M, Hernandez-Reif M, Figueiredo B, Deeds O, Contogeorgos J, Ascencio A (2006) Prenatal paternal depression. Infant Behav Dev 29(4):579-583

Field T, Diego M, Hernandez-Reif M, Figueiredo B, Schanberg S, Kuhn C, Deeds O, Contogeorgos J, Ascencio A (2008) Chronic prenatal depression and neonatal outcome. Int $\mathrm{J}$ Neurosci 118:95-103

Figueiredo B, Pacheco A, Costa R (2007) Depression during pregnancy and the postpartum period in adolescent and adult Portuguese mothers. Arch Womens Ment Health 10:103-109

Gorman LL, O'Hara MW, Figueiredo B, Hayes S, Jacquemain F, Kammerer MH, Klier CM, Rosi S, Seneviratne G, Sutter-Dallay 
AL, TCS-PND Group (2004) Adaptation of the structured clinical interview for DSM-IV disorders for assessing depression in women during pregnancy and post-partum across countries and cultures. Br J Psychiatry Suppl 46:17-23

Heron J, O'Connor TG, Evans J, Golding J, Glover V, ALSPAC Study Team (2004) The course of anxiety and depression through pregnancy and the postpartum in a community sample. J Affect Disord 80:65-73

Huizink AC, Mulder EJ, Robles de Medina PG, Visser GH (2004) Is pregnancy anxiety a distinctive syndrome? Early Hum Dev 79:81-91

INE-Instituto Nacional de Estatística, Portugal (2001) Inquérito à fecundidade e família: resultados definitivos: 1997 [1997 Fertility and Family Survey (FFS)]. INE — Secção de Artes Gráficas, Lisboa

Kammerer M, Taylor A, Glover V (2006) The HPA axis and perinatal depression: a hypothesis. Arch Womens Ment Health 9:187-196

Kammerer M, Marks MN, Pinard C, Taylor A, von Castelberg B, Künzli H, Glover V (2009) Symptoms associated with the DSM IV diagnosis of depression in pregnancy and postpartum. Arch Womens Ment Health 12:135-141

Lee AM, Lam SK, Sze Mun Lau SM, Chong CS, Chui HW, Fong DY (2007) Prevalence, course, and risk factors for antenatal anxiety and depression. Obstet Gynecol 110:1102-1112

Limlomwongse N, Liabsuetrakul T (2006) Cohort study of depressive moods in Thai women during late pregnancy and 6-8 weeks of postpartum using the Edinburgh Postnatal Depression Scale (EPDS). Arch Womens Ment Health 9:131-138

Matthey S, Barnett B, Ungerer J, Waters B (2000) Paternal and maternal depressed mood during the transition to parenthood. $\mathrm{J}$ Affect Disord 60:75-85

Morse CA, Buist A, Durkin S (2001) First time parenthood: influences on pre and postnatal adjustment in fathers and mothers. $\mathrm{J}$ Psychosom Obstet Gynaecol 21:109-120
O'Hara MW, Swain AM (1996) Rates and risk of postpartum depression-a meta-analisys. Int Rev Psychiatry 8:37-54

Perren S, von Wyl A, Bürgin D, Simoni H, von Klitzing K (2005) Depressive symptoms and psychosocial stress across the transition to parenthood: associations with parental psychopathology and child difficulty. J Psychosom Obstet Gynaecol 26:173-183

Ross LE, McLean LM (2006) Anxiety disorders during pregnancy and the postpartum period: a systematic review. J Clin Psychiatry 67:1285-1298

Ross LE, Gilbert Evans SE, Sellers EM, Romach MK (2003) Measurement issues in postpartum depression part 1: anxiety as a feature of postpartum depression. Arch Womens Ment Health 6:51-57

Ross LE, Sellers EM, Gilbert Evans SE, Romach MK (2004) Mood changes during pregnancy and the postpartum period: development of a biopsychosocial model. Acta Psychiatr Scand 109:457-466

Skari H, Skreden M, Malt UF, Dalholt M, Ostensen AB, Egeland T, Emblem R (2002) Comparative levels of psychological distress, stress symptoms, depression and anxiety after childbirth-a prospective population-based study of mothers and fathers. BJOG 109:1154-1163

Spielberger CD, Gorsuch RL, Lushene R, Vagg PR, Jacobs GA (1983) Manual for the state-trait anxiety inventory. STAI (Form Y). Selfevaluation questionaire. Consulting Psychologists Press, Inc, Palo Alto

Teixeira C, Figueiredo B, Conde A, Pacheco A, Costa R (2009) Anxiety and depression during pregnancy in women and men. $\mathrm{J}$ Affect Disord 119:142-148

Wenzel A, Haugen EN, Jackson LC, Robinson K (2003) Prevalence of generalized anxiety at eight weeks postpartum. Arch Womens Ment Health 6:43-49

Wenzel A, Haugen EN, Jackson LC, Brendle JR (2005) Anxiety symptoms and disorders at eight weeks postpartum. J Anxiety Disord 19:295-311 